\title{
Phase dynamics of discrete breathers periodically tunneling in weakly coupled nonlinear chains
}

\author{
Yuriy A. Kosevich \\ Institute of Chemical Physics, Russian Academy of Sciences, 4 Kosygina ul., Moscow 119991, Russia \\ Plekhanov Russian University of Economics, 36 Stremyanny per., Moscow 117997, Russia \\ E-mail: yukosevich@gmail.com
}

Received February 1, 2018, published online May 28, 2018

\begin{abstract}
We present a brief discussion of the phase-coherent dynamics of discrete breathers (intrinsic localized modes) in a system of two weakly coupled nonlinear chains and its comparison with periodic tunneling of quantum particles in a double-well potential and with macroscopic quantum tunneling of two weakly linked Bose-Einstein condensates. We consider the dynamics of relative phase of classically-tunneling discrete breathers in two weakly coupled nonlinear chains and show that the dynamics of the relative phase in the $\pi / 2$ tunneling mode coincides with the experimentally observed dynamics of the relative phase of quantum particles, periodically tunneling in a double-well potential, both for noninteracting and strongly repulsively interacting particles. The observed coincidence demonstrates the correspondence between the dynamics of classical localized excitations in two weakly coupled nonlinear chains and tunneling dynamics of quantum object in the double-well potential. We show that in both $\pi / 2$ and winding tunneling modes the relative phase experiences periodic jumps by $\pi$ in the instants of complete depopulation of one of the weakly coupled chains or potential wells. The connection of the observed phase dynamics with the non-quantum uncertainty principle is discussed.

PACS: $05.45 . Y v$ Solitons;

63.22.-m Phonons or vibrational states in low-dimensional structures and nanoscale materials;

03.75.Lm Tunneling, Josephson effect, Bose-Einstein condensates in periodic potentials, solitons, vortices, and topological excitations;

74.50. $+\mathrm{r}$ Tunneling phenomena; Josephson effects.
\end{abstract}

Keywords: discrete breathers, weakly coupled anharmonic chains, dynamics of relative phase, $\pi / 2$ tunneling mode, winding tunneling mode, periodic tunneling, classical and quantum objects, non-quantum uncertainty principle.

Tunneling through a barrier is purely quantum mechanical phenomenon. On a macroscopic scale it is realized in Josephson effect between two weakly coupled phasecoherent condensates. The latter can be two superconductors separated by a thin barrier [1], two reservoirs of superfluid helium connected by nanoscopic apertures [2,3], or two weakly linked Bose-Einstein condensates (BECs) in a macroscopic double-well potential (single bosonic Josephson junction) [4]. Here we discuss the profound analogy between classical phase-coherent dynamics of discrete breathers (DBs) in two weakly coupled nonlinear chains and tunneling dynamics of quantum objects. Corresponding equations of classical tunneling dynamics of two weakly coupled DBs (wandering breather) were obtained in the first papers in this field [5-7] and were later applied to a great variety of nonlinear mechanical systems [8]. Here we show that the dynamics of the relative phase of DBs in two weakly coupled nonlinear chains in the $\pi / 2$ tunneling mode, first described in [5-7], exactly coincides with the experimentally observed dynamics of the relative phase of quantum particles periodically tunneling in a double-well potential, which is characterized by the abrupt changes (jumps) of the phase between the $\pi / 2$ and $-\pi / 2$ values at the instants when the particles are fully localized in one of the coupled potential wells [9]. The coincidence of the phase dynamics is shown both for noninteracting and strongly repulsively interacting quantum particles. We show that in both $\pi / 2$ and winding tunneling modes, the relative phase experiences periodic jumps by $\pi$ in the instants of complete depopulation of one of the two weakly coupled chains or potential wells.

Nonlinear excitations (solitons, kink-solitons, intrinsic localized modes and discrete breathers) can be created most easily in low-dimensional (1D and quasi-1D) systems [10-20]. Recent experiments have demonstrated the existence of intrinsic localized modes and discrete breathers in various systems such as coupled nonlinear optical waveguides [21], low-dimensional crystals [22], micromechanical oscillator arrays [23,24], antiferromagnetic materials [25], Josephson junction arrays [26,27], optical waveguides and photonic crystals [28], $\alpha$-helices [29], and $\alpha$-uranium [30]. 
Slowly-moving DBs, subsonic and supersonic kinks were also described in $1 \mathrm{D}$ nonlinear chains [15,17,31-37] and quasi-1D polymer crystals [38]. One-dimensional arrays of magnetic or optical microtraps for BECs of ultracold quantum gases with tunneling coupling provide a new field for the studies of coherent nonlinear dynamics in low-dimensional systems [39,40].

Here we discuss another conceptual aspect of classical nonlinear excitations by considering the analogy between dynamics of phase-coherent DBs, either stationary or slowly-moving, in two weakly coupled nonlinear chains and quantum tunneling dynamics of a quantum particle periodically tunneling in a double-well potential [9] and of two weakly linked macroscopic condensates in a single bosonic Josephson junction [5-7]. There are two qualitatively different dynamical regimes of the coupled DBs or intrinsic localized modes, the nonlinear Rabi-like oscillations of the lowamplitude DBs between the chains (tunneling DB), and onechain-localization (nonlinear self-trapping) for the highamplitude DB. These two regimes, which are separated by a separatrix mode with zero rate of energy and excitation exchange, are analogous to the two regimes in nonlinear dynamics of macroscopic Bose-Einstein condensates in a single bosonic Josephson junction [4]. Phase-coherent dynamics of the coupled classical DBs is described by a pair of equations completely similar to that for BECs in a single bosonic Josephson junction $[45,46]$. The considered evolution of the relative phase of two weakly coupled DBs is analogous to the evolution of relative quantum-mechanical phase between two macroscopic condensates, which was directly measured in a single bosonic Josephson junction by means of interference [4]. Moreover, the separatrix in the excitation exchange between macroscopic phase-coherent ensembles of particles in weakly coupled classical chains with "repulsive” nonlinearity can be considered as a nonlinear dynamical model of the reversible interaction-induced superfluid-Mott-insulator transition, which was observed in Bose-Einstein condensate in a lattice with tunneling intersite coupling [41].

\section{Model}

Following [5-7], we consider two linearly coupled identical anharmonic chains (with unit lattice period), which we model with the Fermi-Pasta-Ulam ( $\beta$-FPU) Hamiltonian:

$$
\begin{aligned}
& H=\sum_{n}\left[\sum _ { i = 1 } ^ { 2 } \left[\frac{1}{2} p_{n}^{(i) 2}+\frac{1}{2}\left(u_{n+1}^{(i)}-u_{n}^{(i)}\right)^{2}+\right.\right. \\
& \left.\left.+\frac{1}{4} \beta\left(u_{n+1}^{(i)}-u_{n}^{(i)}\right)^{4}\right]+\frac{1}{2} C\left(u_{n}^{(1)}-u_{n}^{(2)}\right)^{2}\right],
\end{aligned}
$$

where $u_{n}^{(i)}$ is displacement of the $n$th particle from its equilibrium position in the $i$ th chain, $p_{n}^{(i)}=\dot{u}_{n}^{(i)}$ is particle momentum, $\beta$ and $C$ are, respectively, dimensionless intrachain nonlinear and inter-chain linear force constants (in units of intra-chain linear force constant). We assume that the coupling is weak, $C \ll 1$, and do not include the nonlinear inter-chain interaction. Hamiltonian (1) describes, e.g., purely transverse particle motion [15].

We are interested in high-frequency and therefore shortwavelength dynamics of the coupled chains with the frequency close to the top of acoustic phonon band, when the displacements of the nearest-neighbor particles are mainly anti-phase. For this case we introduce continuous envelope-functions $f(x)^{(i)}$ for the particle displacements in the chains, $u_{n}^{(i)}=f(x)^{(i)}(-1)^{n}, \partial f^{(i)} / \partial x \ll 1$, where $x=n$ is a continuous spatial coordinate along the $i$ th chain, which allow us to write partial differencial equations for them, see, e.g., [11,15,32-34]. Then from Hamiltonian (1) we get the following equations for $f(x)^{(i)}, i=1,2$ :

$$
\ddot{f}^{(i)}+(4+C) f^{(i)}+\frac{\partial^{2} f^{(i)}}{\partial x^{2}}+16 \beta f^{(i) 3}-C f^{(3-i)}=0 .
$$

In order to deal with the amplitude and phase of the coupled nonlinear excitations, it is useful to introduce complex wave fields $\Psi(x, t)_{i}$ for each chain, cf. [32]:

$$
f(x, t)^{(i)}=\frac{1}{2}\left[\Psi(x, t)_{i} \exp \left(-i \omega_{m} t\right)+\Psi(x, t)_{i}^{*} \exp \left(i \omega_{m} t\right)\right],
$$

where $\omega_{m}=\sqrt{4+C} \approx 2+C / 4$ is characteristic frequency slightly above the maximal phonon frequency of isolated chains (equal to 2 in each chain in the considered units). Assuming that characteristic frequencies of the fields $\Psi(x, t)_{i}$ are small in comparison with $\omega_{m}$, from Eqs. (2) and (3) we get the following coupled nonlinear-Schrödinger-type equations for $\Psi(x, t)_{i}, i=1,2$ :

$$
-i \omega_{m} \dot{\Psi}_{i}+\frac{1}{2} \frac{\partial^{2} \Psi_{i}}{\partial x^{2}}+6 \beta\left|\Psi_{i}\right|^{2} \Psi_{i}=\frac{C}{2} \Psi_{3-i},
$$

and complex-conjugated equations for $\Psi_{i}^{*}$. Similar Schrödinger-type and nonlinear-Schrödinger-type equations for complex envelope functions of classical displacement fields were obtained in [32,42].

Using Eqs. (4), one can readily show the existence of the following integrals of motion and inter-chain flux:

$$
\begin{gathered}
N_{s}=\int\left[\left|\Psi_{1}\right|^{2}+\left|\Psi_{2}\right|^{2}\right] d x \equiv N_{1}+N_{2} \\
E_{S}=\int\left[\sum_{i=1}^{2}\left(3 \beta\left|\Psi_{i}\right|^{4}-\frac{1}{2}\left|\frac{\partial \Psi_{i}}{\partial x}\right|^{2}\right]-\frac{1}{2} C\left(\Psi_{1} \Psi_{2}^{*}+\Psi_{2} \Psi_{1}^{*}\right)\right] d x \\
P_{S X}=-\frac{i}{2} \int \sum_{i=1}^{2}\left[\Psi_{i} \frac{\partial \Psi_{i}^{*}}{\partial x}-\Psi_{i}^{*} \frac{\partial \Psi_{i}}{\partial x}\right] d x \\
J_{i}=\frac{i C}{2 \omega_{m}} \int\left[\Psi_{i} \Psi_{3-i}^{*}-\Psi_{i}^{*} \Psi_{3-i}\right] d x=-J_{3-i}
\end{gathered}
$$

which describe, respectively, the total number of excitations, total energy, total momentum along the chain axis, 
and total inter-chain flux of excitations (which conserves the total number of them, $\dot{N}_{i}+J_{i}=0$ ), cf. [32]. The existence of these integrals of motion and inter-chain flux demonstrates that the exchange of energy between two coupled nonlinear systems is a coherent phenomenon, which depends, in general, on the initial conditions.

\section{Tunneling dynamics of weakly coupled discrete breathers}

To describe the slowly-moving DB, periodically tunneling between two weakly coupled nonlinear chains with positive (repulsive) anharmonic force constant $\beta$, we assume the following form for the complex fields $\Psi_{1}$ and $\Psi_{2}$ [5-7]:

$$
\begin{aligned}
& \Psi_{1}=\Psi_{\max } \frac{\exp [i(k x-\Omega t)]}{\cosh \left[\kappa_{1}(x-V t)\right]} \cos \Theta \exp \left(-\frac{i}{2} \Delta\right), \\
& \Psi_{2}=\Psi_{\max } \frac{\exp [i(k x-\Omega t)]}{\cosh \left[\kappa_{2}(x-V t)\right]} \sin \Theta \exp \left(\frac{i}{2} \Delta\right),
\end{aligned}
$$

where $\Omega>0$ describes small shift of the DB frequency $\omega$ with respect to $\omega_{m}, V \ll 1$ and $k \ll 1$ are the velocity and wavenumber related with the moving $\mathrm{DB}, \kappa_{i}$ describe inverse localization lengths. Here $\Delta=\Delta(t-k x / \omega)$ stands for the reduced phase of the lattice excitations in the coupled chains, while the parameter $\Theta=\Theta(t-k x / \omega)$ describes the relative population (population imbalance) of the two chains $z=\left(n_{1}-n_{2}\right) /\left(n_{1}-n_{2}\right)=\cos 2 \Theta$, where $n_{i}=\left|\Psi_{1}\right|^{2}$ is local density of excitations in the $i$ th chain, and $n_{1}+n_{2}=$ $=\left|\Psi_{\max }\right|^{2}=$ const.

Parameters $\Delta$ and $\Theta$ determine the inter-chain flux of excitations, cf. Eq. (8):

$$
J_{1}=-J_{2}=\frac{C \Psi_{\max }^{2}}{2 \omega_{m}} \int \frac{\sin 2 \Theta \sin \Delta}{\cosh \left[\kappa_{1}(x-V t)\right] \cosh \left[\kappa_{2}(x-V t)\right]} d x .
$$

The relative phase $\Phi$, which we will compare below with the relative phase in quantum tunneling dynamics, is defined as

$$
\Phi=\arg \left[\frac{\Psi_{2}}{\Psi_{1}}\right]=\arg [\tan \Theta \exp (i \Delta)] .
$$

It is worth underlining that the relative and reduced phases $\Phi$ and $\Delta$ coincide in the case of tunneling dynamics, in which the tunneling object does not reach during its evolution the state of complete depopulation of one of the weakly coupled atomic chains or potential wells, which corresponds to $|z|=1$. Such tunneling dynamics, with equal relative and reduced phases, was realized, e.g., in Ref. 4 for the Bose-Einstein condensate in weakly linked doublewell potential (bosonic Josephson junction).

Using Eqs. (4), (9) and (10), after some algebra we obtain dispersion equations for the introduced parameters,

$$
\Omega=\frac{1}{2 \omega_{m}}\left[3 \beta \Psi_{\text {max }}^{2}-k^{2}-C \frac{\cos \Delta}{\sin (2 \Theta)}\right], V=\frac{\partial \Omega}{\partial k},
$$

$$
\kappa_{1}^{2}=6 \beta \Psi_{\max }^{2}\left\langle\cos ^{2} \Theta\right\rangle, \quad \kappa_{2}^{2}=6 \beta \Psi_{\max }^{2}\left\langle\sin ^{2} \Theta\right\rangle,
$$

and evolution equations for the parameters $\Theta$ and $\Delta$ :

$$
\dot{\Theta}=\frac{C}{2 \omega_{m}} \sin \Delta,
$$

$$
\dot{\Delta}=\frac{3 \beta \Psi_{\max }^{2}}{\omega_{m}} \cos (2 \Theta)+\frac{C}{\omega_{m}} \cos \Delta \cot (2 \Theta) .
$$

Angular brackets in Eq. (14) denote the time-averaged and therefore time-independent quantities, which take into account the possibility of the integration along the trajectory of the slowly-moving DB in the integrals like that given by Eqs. (5)-(8). In the derivation of Eqs. (15) and (16), it was assumed explicitly that the ratio $\frac{\cosh \left[\kappa_{1}(x-V t)\right]}{\cosh \left[\kappa_{2}(x-V t)\right]}$ is equal to one. The latter is valid for small-amplitude DBs with long localization lengths, $\kappa_{1,2} \ll 1$. In this case the above assumption, which is exact for the central region of the DBs, $x-V t \approx 0$, will be (approximately) valid for a large number of particles, which form weakly localized tunneling DB in weakly coupled nonlinear chains. Equations, similar to Eqs. (15) and (16), were derived, e.g., in Refs. 43, 44 for the description of energy exchange between two nonlinear molecules or weakly coupled classical anharmonic oscillators.

Equations (15) and (16) can be written in an equivalent form for the reduced relative phase $\Delta$ and relative population of the two chains $z=\left(n_{1}-n_{2}\right) /\left(n_{1}+n_{2}\right)=\cos 2 \Theta$, which are canonically conjugate, except the point of $|z|=1$, see below:

$$
\begin{gathered}
\dot{z}=-\frac{\partial H_{\text {eff }}}{\partial \Delta}=-\frac{C}{\omega_{m}} \sqrt{1-z^{2}} \sin \Delta, \\
\dot{\Delta}=\frac{\partial H_{\text {eff }}}{\partial z}=\frac{3 \beta \Psi_{\max }^{2}}{\omega_{m}} z+\frac{C}{\omega_{m}} \frac{z}{\sqrt{1-z^{2}}} \cos \Delta,
\end{gathered}
$$

with the following effective Hamiltonian (which has the dimension of frequency):

$$
H_{\mathrm{eff}}=\frac{3 \beta \Psi_{\max }^{2}}{2 \omega_{m}} z^{2}-\frac{C}{\omega_{m}} \sqrt{1-z^{2}} \cos \Delta
$$

The very same equations were derived in $[45,46]$ in connection with theoretical studies, based on macroscopic quantum Gross-Pitaevskii equation, of coherent atomic tunneling and coherent oscillations between two weakly coupled Bose-Einstein condensates, which were later used in the analysis of the experimental realization of a single bosonic Josephson junction [4]. In our case, Eqs. (17) and (18) describe the exchange of lattice excitations between the chains rather than atomic tunneling. One can consider such excitation exchange as a classical counterpart of macroscopic quantum tunneling dynamics.

It is noteworthy that the evolution equations, similar to Eqs. (15) and (16), describe the dynamics of two weakly 
coupled identical nonlinear oscillators [43,44]. Therefore the tunneling intrinsic localized modes or discrete breathers can be considered as weakly coupled phase-coherent nonlinear macroscopic oscillators. Since nonlinear macroscopic oscillators can posses nonlinearity of both signs, the evolution equations (15) and (16) and their derivatives, Eqs. (23) below, can be considered in general for both positive and negative sign of the anharmonic force constant $\beta$.

Equations (15) and (16) can be solved analytically for the given initial conditions. [For the weak coupling, in the following we assume $\omega_{m}=2$ ]. Using the ansatz,

$$
\cos \Delta=A(t) / \sin (2 \Theta) \text {, }
$$

where $A=0$ for $\Theta=0$, we get from Eqs. (15) and (16):

$$
\dot{A}=-\left(3 \beta \Psi_{\text {max }}^{2} / C\right) \sin (4 \Theta) \dot{\Theta} .
$$

We are seaking for the solution of Eqs. (15) and (16) with the initial condition $\Theta(0)=0$, which corresponds to zero complex field (complex wave function) $\Psi_{2}$ in the second chain at $t=0$. For $\Theta(0)=0$ and $A(0)=0$, we obtain the following exact solution of Eqs. (15) and (16):

$$
\begin{gathered}
A=-\frac{3 \beta \Psi_{\max }^{2}}{2 C} \sin ^{2}(2 \Theta), \\
\cos \Delta=-\frac{3 \beta \Psi_{\max }^{2}}{2 C} \sin (2 \Theta)=-\frac{3 \beta \Psi_{\max }^{2}}{2 C} \sqrt{1-z^{2}},
\end{gathered}
$$

which corresponds to $\Delta(0)=\pi / 2 \bmod \pi$. Then Eqs. (15) and (16) can be reduced to the equations

$$
\dot{\Theta}=\frac{C}{4} \sin \Delta, \quad \dot{\Delta}=\frac{3 \beta \Psi_{\max }^{2}}{4} \cos (2 \Theta),
$$

which will be solved below with the initial conditions $\Theta(0)=0$ and $\Delta(0)=\pi / 2$.

Finally, from Eqs. (22) and (23) we get the following two equivalent pendulum equations:

$$
\ddot{\delta}+\frac{C^{2}}{4} \sin \delta=0
$$

for $\delta=2 \Delta-\pi$, and

$$
\ddot{\Xi}+\Omega_{0}^{2} \sin \Xi=0
$$

for $\Xi=4 \Theta$, where $\Omega_{0}=3 \beta \Psi_{\max }^{2} / 4$.

The pendulum equation can be solved exactly, see, e.g., Refs. 44, 47. We are interested in the solution of Eq. (24) with the initial conditions $\delta(0)=0$ and $\dot{\delta}(0)=\frac{3}{2} \beta \Psi_{\text {max }}^{2}$. The corresponding initial conditions for $\Xi$ in Eq. (25) are $\Xi(0)=0$ and $\dot{\Xi}(0)=C$.

The important property of Eqs. (23) and of corresponding pendulum equations (24) and (25) is the existence of two qualitatively different dynamical regimes of excitation and energy exchange, which have a separatrix realized for the condition $|S|=1$, where $S=3 \beta \Psi_{\text {max }}^{2} /(2 C)$ is the nonlinearity-over-coupling parameter.
For $|S| \ll 1$, Eqs. (23) describe the mode, in which the parameter $\Theta$ linearly grows with the "running" time $\tilde{t} \equiv$ $\equiv t-k x / \omega, \omega=2$ :

$$
\begin{gathered}
\Theta \approx \tilde{C} \frac{\tilde{t}}{4}+\frac{9 \beta^{2} \Psi_{\max }^{4}}{64 \tilde{C}^{2}} \sin (\tilde{C} \tilde{t}), \\
\Delta \approx \frac{\pi}{2}+\frac{3 \beta \Psi_{\max }^{2}}{2 \tilde{C}} \sin \left(\tilde{C} \frac{\tilde{t}}{2}\right), \\
z \approx \cos \left(\tilde{C} \frac{\tilde{t}}{2}\right), \\
\tilde{C}=C-\frac{\Omega_{0}^{2}}{C}=C-\frac{9 \beta^{2} \Psi_{\max }^{4}}{16 C} .
\end{gathered}
$$

In this regime, $\Theta$ spans the full range from 0 to $2 \pi$, which corresponds to the total energy exchange between the nonlinear chains and therefore to the DB, periodically tunneling between the two chains, when the reduced phase $\Delta$ is close to $\pi / 2$. We call the excitation exchange in this regime as the $\pi / 2$ tunneling mode of DB. According to Eq. (11), the frequency of periodic inter-chain flux of excitations and energy in this mode, $J_{1} \propto C \Psi_{\text {max }} \sin 2 \Theta \sin \Delta \approx C \Psi_{\text {max }} \sin (\tilde{C} \tilde{t} / 2)$, is determined by the inter-chain coupling $\tilde{C} / 2$, reduced by the nonlinearity parameter $\beta \Psi_{\max }^{2}$, see Eq. (29).

The relative phase $\Phi$ is defined by Eq. (12) and its time evolution in the $\pi$ / 2 tunneling mode, described by Eqs. (26) and (27), is presented in Fig. 1 for different values of the nonlinearity-over-coupling parameter $S=3 \beta \Psi_{\text {max }}^{2} /(2 C)$.

Figure 1 clearly shows that there are abrupt changes (jumps) of the relative phase $\Phi$ by $\pi$, between $\pi / 2$ and $-\pi / 2$, at each instant when the DB is located completely in one of the coupled chains and the relative population is equal to unit in modulus, $|z|=1$. According to Eq. (27), the interval between such instants in the weakly-nonlinear limit is given by $2 \pi / C$. In the case of vanishing nonlinearity, the time evolution of the relative phase $\Phi$ coincides exactly with the experimentally observed dynamics of the relative phase of the tunneling quantum particles in a doublewell potential [9]. The jumps of the relative phase at the instants of $|z|=1$ are related with the uncertainty of the phase when the particle wave function is exactly zero in one of the coupled potential wells or chains, $\Psi_{i}=0$ either for $i=1$ or $i=2$, see Eqs. (9) and (10). On the other hand, the abrupt change of the relative phase can be related with the (non-quantum) uncertainty principle: the variables $\mathrm{Z}$ and $\Phi$ are canonically conjugate, see Eqs. (17) and (18), and therefore cannot be measured simultaneously because the product of their uncertainties $\Delta z$ and $\Delta \Phi$ is bounded from below by the inequality $\Delta z \cdot \Delta \Phi \geq 1 / 2$. At the instants of $|z|=1$, the inter-chain tunneling current is zero, $\dot{z}=0$, see (11), and therefore the relative population $z$ and correspondingly the location of the $\mathrm{DB}$ or quantum particle is well defined, which makes the value of $\Phi$ be uncertain at these instants. 

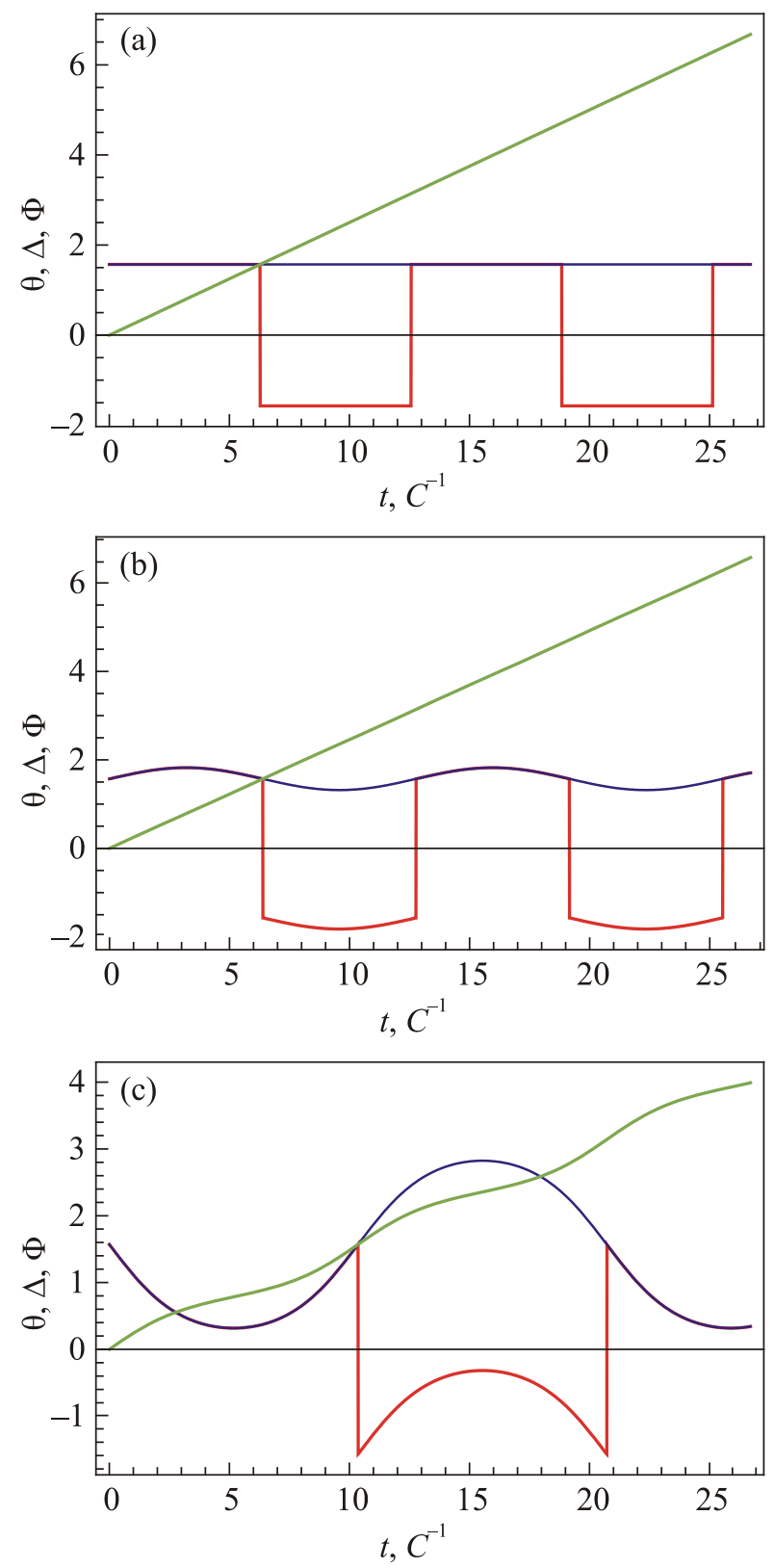

Fig. 1. (Color online) Time evolution of parameter $\Theta$ (green lines), reduced phase $\Delta$ (blue lines) and relative phase $\Phi$ (red lines) of two DBs in the $\pi / 2$ tunneling mode between two weakly coupled nonlinear chains: (a) for $S=0$, (b) for $S=0.25$, and (c) $S=-0.95$. Dynamics of the relative phase is described by Eqs. (12), (26) and (27), time is measured in $C^{-1}$.

Equations (28) and (29) also predict substantial reduction of the tunneling frequency by the nonlinearity, which in turn can be compared with the observed transition from the high first-order (single-particle) to low second-order (two-particle co-tunneling) tunneling rates of quantum particles in the double-well potential, caused by the repulsion between particles [9]. Figure 1(c) shows phase dynamics in the $\pi / 2$ tunneling mode, which describes periodic complete depopulation of the coupled chains for $S=-0.95$, which corresponds to the $\beta$-FPU Hamiltonian (1) with negative $\beta$. This value of the nonlinearity-over-coupling parameter $S$ can be put in line with strong repulsion between periodically tunneling quantum particles with positive masses in the experiments [9] because lattice excitations in acoustic phonon band in monatomic chain with nearest-neighbor interactions possess negative effective mass [32,36]. Nonlinear excitations in the $\beta$-FPU model with negative $\beta$ are the dark lattice solitons [15,16] (and tunneling dark lattice solitons in two weakly coupled chains), which are formed due to effective repulsion between the quasiparticles with negative effective mass. In the tunneling dark lattice solitons in coupled $\beta$-FPU chains, the factors $\Psi_{\max } \exp [i(k x-\Omega t)] / \cosh \left[\kappa_{i}(x-V t)\right]$ with $\Omega>0$ in the complex fields (9) and (10) are replaced by the factors $\Psi_{\max } \exp [i(k x-\Omega t)] \tanh \left[\kappa_{i}(x-V t)\right]$ with $\Omega<0, i=1,2$, see, e.g., [15]. The origin of dark lattice solitons in the $\beta$-FPU chain with negative $\beta$ can in turn be put in line with the origin of the dark solitons and vortices in Bose-Einstein condensate, which are formed due to the repulsion between quantum particles with positive masses, which is described by the nonlinear term $g|\Psi|^{2} \Psi$ with positive $g$ in the Gross-Pitaevskii equation [48]. Figure 1(c) demonstrates the dynamics of relative phase $\Phi$ with reduced tunneling rate, which is similar to the phase dynamics observed in the experiments [9] in the case of slow second-order co-tunneling of quantum particles with strong repulsion.

Figure 1 also shows that both the reduced phase $\Delta$ and parameter $\Theta$ are continuous and single-valued functions (of time) and the wave functions (9) and (10) of the displacement fields, which are determined by these parameters, are the single-valued functions as it is required by quantum mechanics [49], and the jumps by $\pi$ of the relative phase $\Phi$, which is determined by (12), do not make the wave functions ambiguous functions. The continuity of the $\Delta$ and $\Theta$ functions is confirmed by the existence of the second-order equations (24) and (25) for these functions, which provide description of the tunneling dynamics, equivalent to that given by Eqs. (23). Since both the classically tunneling DBs in a system of two weakly coupled nonlinear chains and the quantum particles, periodically tunneling in a double-well potential, can be described with the use of the canonically conjugate relative population $\mathrm{Z}$ and reduced phase $\Delta$ (and relative phase $\Phi$ ), there is clear coincidence of the phase dynamics and the correspondence between periodic tunneling of classical and quantum objects.

The phase dynamics in the $\pi / 2$ tunneling mode can also be compared with the phase dynamics in the quantum Rabi oscillations, see, e.g., [50]. Here, for the superposition of two states

$$
\Psi(t)=a_{1}(t)|1\rangle+a_{2}(t)|2\rangle
$$

with equal on-site energies $E_{1}=E_{2} \equiv E$, Schrödinger equations for the complex amplitudes $a_{1}$ and $a_{2}$ are the following:

$$
\begin{gathered}
i \dot{a}_{1}=E a_{1}-\gamma a_{2}, \\
i \dot{a}_{2}=E a_{2}-\gamma a_{1},
\end{gathered}
$$


where parameter $\gamma$ is the coupling matrix element between the states, and $\hbar=1$. For the initial conditions $a_{1}(0)=1$ and $a_{2}(0)=0$ and the value of the coupling parameter $\gamma=C / 4$, which corresponds to Eqs. (4), Eqs. (31) and (32) have the solution

$$
\begin{gathered}
a_{1}=\cos (\gamma t) \exp (-i E t)=\cos (C t / 4) \exp (-i E t), \\
a_{2}=i \sin (\gamma t) \exp (-i E t)=i \sin (C t / 4) \exp (-i E t),
\end{gathered}
$$

which exactly corresponds to the $\pi / 2$ tunneling mode, described by Eqs. (26) and (27) in the linear case $S=0$ for $\Theta=C t / 4$ and $\Delta=\pi / 2$. [In the system of two weakly coupled atomic chains, the linear case $S=0$ corresponds to two weakly coupled identical harmonic chains, with $C \ll 1$ and equal final lengths, when the $\pi / 2$ tunneling mode describes the periodic inter-chain tunneling of the confined harmonic vibrational mode at the top of acoustic band of the chains, with eigenfrequency $\omega=\omega_{m} \approx 2$.] Therefore the $\pi / 2$ tunneling mode for $0<|S|<1$ can be considered as the nonlinear Rabi-like oscillations of excitation population in two coupled anharmonic chains, in which the complex wave functions $\Psi_{1}$ and $\Psi_{2}$ in the ansatz (9) and (10) play role of the complex amplitudes $a_{1}$ and $a_{2}$ in the superposition of two states (30). The $\pi / 2$ tunneling mode, which is described by the single-valued wave function in accordance with the requirement of quantum mechanics [49], can also be realized in two weakly linked macroscopic quantum Bose-Einstein condensates.

The separatrix, realized for $|S|=1$ and $H_{\text {eff }}=C / \omega_{m}$, is characterized by infinite oscillation period of the physical pendulum (24) or (25), which corresponds to the infinite period of inter-chain energy exchange. For the considered initial conditions, the separatrix is described by the following solution of Eqs. (23):

$$
\begin{gathered}
\Theta=\arctan \left[\exp \left(\frac{C}{2} t\right)\right]-\frac{\pi}{4}, \Delta=2 \arctan \left[\exp \left(\frac{C}{2} t\right)\right], \\
\Theta(0)=0, z(0)=1, z(\infty)=0, \Delta(0)=\frac{\pi}{2}, \Delta(\infty)=\pi .
\end{gathered}
$$

There is no difference between the reduced $\Delta$ and relative $\Phi$ phases in the separatrix mode for $t>0$ because the state of complete depopulation of the one of the coupled chains $|z|=1$ is reached only at $t=0$, see Eq. (36). The flux of the interchain excitation exchange is zero at the separatrix for $t \rightarrow \infty$ : $J_{i}=0$ since $\sin (\Delta(\infty))=0$, see Eq. (11).

Beyond the separatrix, in the limit of $|S| \gg 1$, one can obtain the following expressions for the evolution of $\Theta, \Delta$ and $z$ with the running time $\tilde{t} \equiv t-k x / \omega, \omega=2$ :

$$
\begin{gathered}
\Theta \approx \frac{C}{3 \beta \tilde{\Psi}_{\max }^{2}} \sin \left(\frac{3}{4} \beta \tilde{\Psi}_{\max }^{2} \tilde{t}\right), \\
\Delta \approx \frac{\pi}{2}+\frac{3}{4} \beta \tilde{\Psi}_{\max }^{2} \tilde{t}+\frac{C^{2}}{18\left(\beta \tilde{\Psi}_{\max }^{2}\right)^{2}} \sin \left(\frac{3}{2} \beta \tilde{\Psi}_{\max }^{2} \tilde{t}\right),
\end{gathered}
$$

$$
\begin{gathered}
z \approx 1-\frac{2 C^{2}}{9\left(\beta \tilde{\Psi}_{\max }^{2}\right)^{2}} \sin ^{2}\left(\frac{3}{4} \beta \tilde{\Psi}_{\max }^{2} \tilde{t}\right), \\
\beta \tilde{\Psi}_{\max }^{2}=\beta \Psi_{\max }^{2}-\frac{C^{2}}{9 \beta \Psi_{\max }^{2}} .
\end{gathered}
$$

This dynamical regime corresponds to the asymmetric nonlinear mode (known, e.g., for two coupled nonlinear waveguides [51-53]), in which one system, here is chain 1, carries almost all vibrational energy while the other is almost at rest. This excitation-exchange regime can be called as the winding tunneling mode of DB because of linear increase in time (winding up) of the reduced phase $\Delta$, see Eq. (38) and Fig. 2. As one can see in Fig. 2, the relative phase $\Phi$ experiences periodic jumps by $\pi$, with the period of $2 \pi /|S|$. The time instants of the relative-phase jumps coincide with the instants of complete depopulation of one of the coupled chains, when $|z|=1$, similar to the case of the phase jumps by $\pi$ in the $\pi / 2$ tunneling mode shown in Fig. 1. As one can see in Figs. 1 and 2, both the reduced phase $\Delta$ and parameter $\Theta$ are continuous and single-valued functions in the $\pi / 2$ and winding tunneling modes, which makes the wave functions (9) and (10) of the displacement

(a)

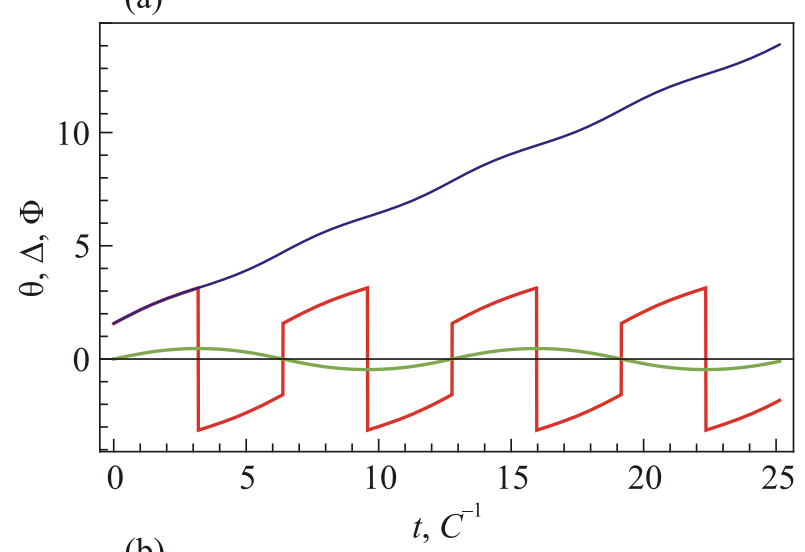

(b)

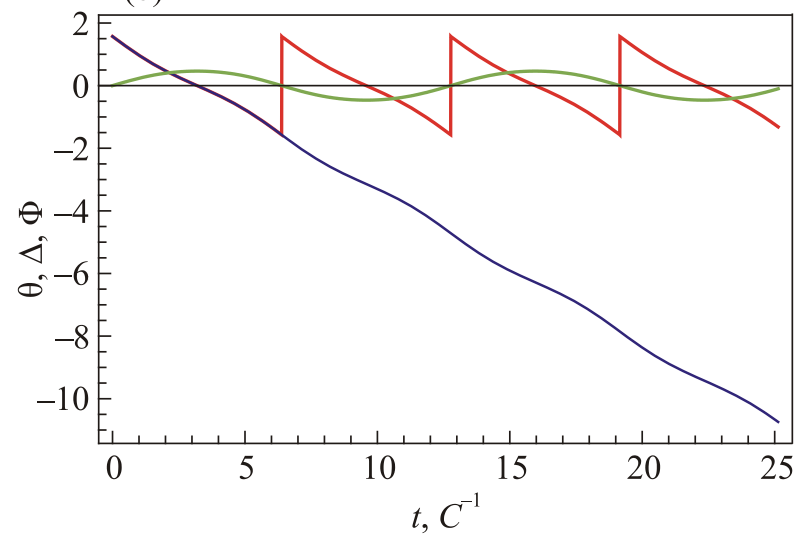

Fig. 2. (Color online) Time evolution of parameter $\Theta$ (green lines), reduced phase $\Delta$ (blue lines) and relative phase $\Phi$ (red lines) of two DBs in the winding tunneling mode between two weakly coupled nonlinear chains: (a) for $S=1.25$, (b) for $S=-1.25$. Dynamics of the relative and reduced phases is described by Eqs. (12), (37) and (38), time is measured in $C^{-1}$. 
fields be the single-valued functions as it is required by quantum mechanics [49]. In the winding tunneling mode, the energy exchange between the coupled chains is relatively weak and gradually decreases with the increase of the parameter $|S|$ beyond the separatrix value of $|S|=1$. The frequency of periodic inter-chain flux of excitations and energy in this mode, $J_{1} \propto C \Psi_{\text {max }} \sin 2 \Theta \sin \Delta \propto$ $\propto\left(C^{2} / \beta \Psi_{\max }\right) \sin \left(3 \beta \tilde{\Psi}_{\max }^{2} \tilde{t} / 2\right)$, is determined by the nonlinearity parameter $3 \beta \tilde{\Psi}_{\max }^{2} / 2$, reduced by the interchain coupling $C$, see Eq. (40).

The two dynamical regimes of energy and excitation exchange between DBs in two weakly coupled nonlinear chains, given by Eqs. (26), (27) and (37), (38), are analogous, respectively, to anharmonic Josephson-like oscillations and nonlinear self-trapping, which were detected in a single bosonic Josephson junction [4].

It is worth mentioning that the form and frequency of a stationary or slowly-moving DB in an isolated chain can be obtained only in the winding tunneling (self-trapping) mode, in which one can consider the limit of $C \rightarrow 0$. Indeed, according to Eq. (37), in this limit one has $\Theta=0$ in Eqs. (9) and (10) and DB frequency is obtained from Eqs. (9), (13), (22) and (38), and is equal to

$$
\omega=2+\Omega+\partial(\Delta / 2) / \partial t=2+\frac{3}{2} \beta \Psi_{\max }^{2}-\frac{k^{2}}{4} .
$$

This expression for the DB frequency exactly coincides with the known expression for a single stationary or slowly-moving DB in the small-amplitude limit, see, e.g., [5-7,11,32]. This coincidence confirms the correct choice of the distribution of the reduced phase $\Delta$ between the tunneling objects, given by $-i \Delta / 2$ and $i \Delta / 2$ in the exponents in Eqs. (9) and (10), similar to that in the superposition of two states in quantum mechanics [50]. It is important to underline that to get this expression for $\omega$, one has to take explicitly into account in Eq. (9) the linear increase in time (winding up) of the reduced relative phase $\Delta$ in the self-trapping mode, given by Eq. (38). The winding up of the relative phase of two weakly coupled macroscopic BECs in the nonlinear self-trapping mode has been directly measured in a single bosonic Josephson junction [4]. This finding gives us an additional argument in favor of the profound similarity between macroscopic tunneling quantum dynamics and phase-coherent dynamics of weakly coupled discrete breathers.

\section{Conclusions}

In conclusion, we have presented analytical description of two qualitatively different regimes of energy exchange between phase-coherent discrete breathers (intrinsic localized modes) in two weakly linked nonlinear chains. These regimes have a profound analogy, and are described by a similar pair of equations, to the anharmonic Josephson-like oscillations and nonlinear self-trapping, which were ob- served in a single bosonic Josephson junction. We show that the dynamics of the relative phase in the $\pi / 2$ tunneling mode coincides with the experimentally observed dynamics of the relative phase of quantum particles, periodically tunneling in a double-well potential, both for noninteracting and strongly repulsively interacting particles. The $\pi / 2$ tunneling mode can also be observed in two weakly linked BoseEinstein condensates. The observed coincidence demonstrates the correspondence between the dynamics of classical localized excitations in two weakly coupled nonlinear chains and tunneling dynamics of quantum particles in a double-well potential. In both $\pi / 2$ and winding tunneling modes, the relative phase $\Phi$ experiences periodic jumps by $\pi$ in the instants of complete depopulation of one of the two weakly coupled atomic chains or potential wells. The connection of the observed phase dynamics with the nonquantum uncertainty principle is discussed. The obtained dispersion and evolution equations, together with the wave functions of the coupled nonlinear excitations, can be applied to the tunneling macroscopic Bose-Einstein condensate, moving along two weakly linked bosonic waveguides, to classically-tunneling phase-coherent discrete breathers (intrinsic localized modes) in two weakly linked macromolecules, $\alpha$-helices or DNA, and to electron-phonon and exciton-phonon polarons in two weakly coupled polymer molecules or semiconductor waveguides.

\section{Acknowledgment}

The author has discussed with Arnol'd Markovich Kosevich the first papers in this field [5-7] before their publication. Arnol'd Markovich was interested in the idea of discrete breathers and intrinsic localized modes, periodically tunneling between two weakly coupled nonlinear chains, and the author is grateful to A.M. for his interest and helpful discussions.

This work was supported by the Federal Agency of Scientific Organizations of Russia (Research Topic 00822014-0013, No. AAAA-A17-117042510268-5).

1. B.D. Josephson, Phys. Lett. 1, 251 (1962).

2. S.V. Pereverzev, A. Loshak, S. Backhaus, J.C. Davis, and R.E. Packard, Nature (London) 388, 449 (1997).

3. K. Sukhatme, Y. Mukharsky, T. Chui, and D. Pearson, Nature (London) 411, 280 (2001).

4. M. Albiez, R. Gati, J. Fölling, S. Hunsmann, M. Cristiani, and M.K. Oberthaler, Phys. Rev. Lett. 95, 010402 (2005).

5. Yu.A. Kosevich, L.I. Manevitch, and A.V. Savin, J. Phys.: Conf. Series 92, 012093 (2007).

6. Yu.A. Kosevich, L.I. Manevitch, and A.V. Savin, Phys. Rev. E 77, 046603 (2008).

7. Yu.A. Kosevich, L.I. Manevitch, and A.V. Savin, J. Sound Vibr. 322, 524 (2009).

8. L.I. Manevitch, A. Kovaleva, V. Smirnov, Y. Starosvetsky, Nonstationary Resonant Dynamics of Oscillatory Chains and Nanostructures, Springer, Singapore (2018). 
9. S. Foelling, S. Trotzky, P. Cheinet, M. Feld, R. Saers, A. Widera, T. Mueller, and I. Bloch, Nature (London) 448, 1029 (2007).

10. N.J. Zabusky and M.D. Kruskal, Phys. Rev. Lett. 15, 240 (1965).

11. A.M. Kosevich and A.S. Kovalev, Sov. Phys. JETP 40, 891 (1974).

12. A.S. Dolgov, Sov. Phys. Solid State 28, 907 (1986).

13. A.J. Sievers and S. Takeno, Phys. Rev. Lett. 61, 970 (1988).

14. J.B. Page, Phys. Rev. B 41, 7835 (1990).

15. Yu.A. Kosevich, Phys. Rev. B 47, 3138 (1993); ibid. 48, 3580E (1993).

16. Yu.A. Kosevich, Phys. Lett. A 173, 257 (1993); ibid. 175, 466 (1993).

17. Yu.A. Kosevich, Phys. Rev. Lett. 71, 2058 (1993).

18. S. Aubry, Physica (Amsterdam) 103D, 201 (1997).

19. S. Flach and C.R. Willis, Phys. Rep. 295, 181 (1998).

20. S. Flach and A.V. Gorbach, Phys. Rep. 467, 1 (2008).

21. H.S. Eisenberg, Y. Silberberg, R. Morandotti, A.R. Boyd, and J.S. Aitchison, Phys. Rev. Lett. 81, 3383 (1998).

22. B.I. Swanson, J.A. Brozik, S.P. Love, G.F. Strouse, A.P. Shreve, A.R. Bishop, W.-Z. Wang, and M.I. Salkola, Phys. Rev. Lett. 82, 3288 (1999).

23. M. Sato, B.E. Hubbard, A.J. Sievers, B. Ilic, D.A. Czaplewski, and H.G. Craighead, Phys. Rev. Lett. 90, 044102 (2003).

24. M. Sato, B.E. Hubbard, and A.J. Sievers, Rev. Mod. Phys. 78, 137 (2006).

25. M. Sato and A.J. Sievers, Nature (London) 432, 486 (2004).

26. E. Trias, J.J. Mazo, and T.P. Orlando, Phys. Rev. Lett. 84, 741 (2000).

27. P. Binder, D. Abraimov, A.V. Ustinov, S. Flach, and Y. Zolotaryuk, Phys. Rev. Lett. 84, 745 (2000).

28. D.K. Campbell, S. Flach, and Y.S. Kivshar, Phys. Today 57, 43 (2004).

29. J. Edler, R. Pfister, V. Pouthier, C. Falvo, and P. Hamm, Phys. Rev. Lett. 93, 106405 (2004).

30. M.E. Manley, M. Yethiraj, H. Sinn, H.M. Volz, A. Alatas, J.C. Lashley, W.L. Hults, G.H. Lander, and J.L. Smith, Phys. Rev. Lett. 96, 125501 (2006).

31. D. Chen, S. Aubry, and G.P. Tsironis, Phys. Rev. Lett. 77, 4776 (1996).

32. Yu.A. Kosevich and S. Lepri, Phys. Rev. B 61, 299 (2000).

33. Yu.A. Kosevich and G. Corso, Physica (Amsterdam) 170D, 1 (2002).

34. Yu.A. Kosevich, R. Khomeriki, and S. Ruffo, Europhys. Lett. 66, 21 (2004).
35. J.F.R. Archilla, Yu.A. Kosevich, N. Jimenez, V.J. SánchezMorcillo, and L.M. García-Raffi, Phys. Rev. E 91, 022912 (2015).

36. Yu.A. Kosevich, J. Phys.: Conf. Series 833, 012021 (2017).

37. A. Mehrem, L.J. Salmerón-Contreras, N. Jiménez, V.J. Sánchez-Morcillo, R. Picó, L.M. García-Raffi, J.F.R. Archilla, and Yu.A. Kosevich, Nonlinear Systems, Vol. 2: Nonlinear Phenomena in Biology, Optics and Condensed Matter, J.F.R. Archilla, F. Palmero, C.M. Lemos, B. Sánchez-Rey, and J. Casado-Pascual (eds.), Springer, Cham (2018), p. 261.

38. A.V. Savin and L.I. Manevitch, Phys. Rev. B 67, 144302 (2003).

39. B.P. Anderson and M.A. Kasevich, Science 282, 1686 (1998).

40. T. Kinoshita, T. Wenger, and D.S. Weiss, Nature (London) 440, 900 (2006).

41. M. Greiner, O. Mandel, T. Esslinger, T.W. Hänsch, and I. Bloch, Nature (London) 415, 39 (2002).

42. Yu.A. Kosevich, L.I. Manevitch, and E.L. Manevitch, Usp. Fiz. Nauk 180, 1331 (2010) [Phys. Usp. 53, 1281 (2010)].

43. A. A. Ovchinnikov, Zh. Eksp. Teor. Fiz. 57, 263 (1969) [Sov. Phys. JETP 30, 147 (1970)].

44. A. M. Kosevich and A. S. Kovalev, Introduction to Nonlinear Physical Mechanics, Naukova Dumka, Kiev (1989) (in Russian).

45. A. Smerzi, S. Fantoni, S. Giovanazzi, and S.R. Shenoy, Phys. Rev. Lett. 79, 4950 (1997).

46. S. Raghavan, A. Smerzi, S. Fantoni, and S.R. Shenoy, Phys. Rev. A 59, 620 (1999).

47. R. Z. Sagdeev, D. A. Usikov, and G. M. Zaslavsky, Nonlinear Physics: From the Pendulum to Turbulence and Chaos, Harwood Acad. Publ., New York (1988).

48. L.P. Pitaevskii and S. Stringari, Bose-Einstein Condensation, Clarendon Press, Oxford (2003).

49. L.D. Landau and E.M. Lifshitz, Quantum Mechanics. NonRelativistic Theory, Pergamon Press, Oxford (1977).

50. C. Cohen-Tannoudji, B. Diu, and F. Laloë, Quantum Mechanics, Vol. 1, Chapter IV, Wiley and Hertmann, Paris (1977).

51. S.M. Jensen, IEEE J. Quantum Electron. QE-18, 1580 (1982).

52. N. Akhmediev and A. Ankiewicz, Phys. Rev. Lett. 70, 2395 (1993).

53. I.M. Uzunov, R. Mushall, M. Golles, Yu.S. Kivshar, B.A. Malomed, and F. Lederer, Phys. Rev. E 51, 2527 (1995). 